\title{
A SURVEY ON ENERGY EFFICIENCY FOR WIRELESS MESH NETWORK
}

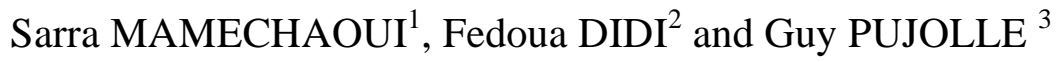 \\ ${ }^{1,2}$ STIC Laboratory, Abou Bekr Belkaid University Tlemcen, Algeria \\ \{sarra.mamechaoui,f_didi\}@mail.univ-tlemcen.dz \\ ${ }^{3}$ Pierre et Marie Curie University Paris 6, FRANCE \\ Guy.Pujolle@lip6.fr
}

\begin{abstract}
Reduction of CO2 emissions is a major global environmental issue. Over the past few years, wireless and mobile communications are becoming increasingly popular with consumers. The Most popular kind of wireless access is known as Wireless Mesh Networks (WMNs) that provide wireless connectivity through lot cheaper and more supple backhaul infrastructure relative to wired solutions. Wireless Mesh Network $(W M N)$ is a new emerging technology which has been adopted as the wireless internetworking solution for the near future. Due to higher energy consumption in the information and communication technology (ICT) industries, and which would have an impact on the environment, energy efficiency has become a key factor to evaluate the performance of a communication network.
\end{abstract}

This paper primarily focuses on the classification layer the greatest existing approaches devoted to the conservation of energy. It is also discussing the most interesting works on energy saving in WMNs networks.

\section{KEYWORDS}

Wireless mesh network (WMN), 802.11s, energy saving, routing protocols, MAC protocols.

\section{INTRODUCTION}

Worldwide energy consumption is now one of the main concerns experienced by governments throughout the world, Due to its significant environmental footprint and the eventual exhaustion [1]. Into a not too far future, the main conventional energy sources shall be replaced with another source using a source of sustainable energy such as solar or wind energy. Green networking has recently attracted a much of attention, including a reflection on the way in which networks are constructed and operated in such a way that not only cost and performance are taken into account, but also their energy consumption and carbon footprint [2].

Applying green networking to wireless mesh networks (WMN) has rarely been described in the literature. Wireless Mesh Network (WMN) [3] is a new emerging technology which has been adopted as the wireless internetworking solution for the near future. Characteristics of WMN like rapid deployment and self configuration make WMN appropriate for transient on-demand network deployment scenarios such as the disaster recovery process, hard-to-wire buildings, conventional networks and friendly terrains. Wireless Mesh Network is now being extensively used as a cost-effective means for coverage extension and backhaul relaying between IEEE 802.11 access points. 
This solution aims to implement mobility in wireless mesh networks. Indeed, Mesh technology has captured the interest of academic research and industry, because of its ability to satisfy both the requirements of Internet Service Providers (ISPs) and wireless users. The Figure 1 show an example of a WLAN Mesh Network architecture [4] that consist of nodes called Mesh points mesh (Mesh Point MP) which only relay traffic but do not provide wireless coverage for mobile station (MSs). These MPs are using the services of wireless mesh network (WLAN Mesh) to communicate with other MPs in the network. They can act as an access point (Mesh Access Point MAP) or gateway (Mesh Point Portal: MPP) whose aim to integrate WMNs with various existing wireless networks such as cellular systems, wireless sensor networks, wireless-fidelity (Wi-Fi) [5] systems, worldwide inter-operability for microwave access (WiMAX) [6].

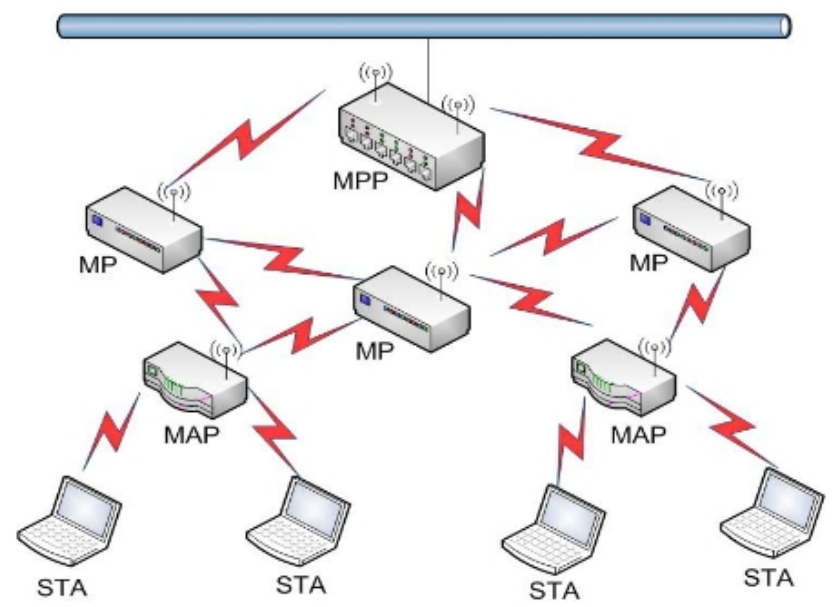

Figure 1: WLAN Mesh Networks architecture.

Due to increased energy consumption in the information and communication technology (ICT) industries, and its consequent environmental effects, energy efficiency has become a key factor to evaluate the performance of a communication network. In WMNs, the resources of Wireless Access Networks are, over long periods of time, under-employment, since only a few percent of the capacity of these devices is actually used and results in high energy waste [7]. This means that the power consumption does not decrease when traffic is light and it might be possible to save large amounts of power while turning off unnecessary network components.

In this context and in order to build green WMNs, it is important to design energy efficient planning and management strategies. This Paper addresses the incorporation of energy conservation at three layers lower of the protocol stack for wireless mesh networks. The remainder of this paper is organized as follows. Section 2 introduces the protocols that save energy in the network layer. Section 3 describes work dealing with energy efficient protocols in the MAC layer of wireless mesh networks, and power conserving protocols in the physical layer are addressed in section 4 . Finally, Section 5 provides a summary and concludes the paper.

\section{ENERGY CONSERVATION IN NETWORK LAYER}

The network layer is responsible for network self-configuration and data routing. To configure the network topology, the network layers choose an appropriate mode for a node and determine the most suitable neighbors with which to associate and form communication links. 
Routing protocols enable a network for making dynamic adjustments to its conditions such decisions are not required to be specific and static. Although the routing protocols functions are plausible, they still encounter difficulties in energy efficiency.

The routing protocols can be split into three categories: Table-driven, the Demand-driven and the Hybrid routing protocols. Routing protocols which use table-driven features are also known as proactive routing protocol, each node maintains one or more tables that have routing information to all other nodes in the network. In this protocol, all nodes update each other on the same network and further update their tables to maintain a consistent and up-to-date view of the network. Demand-driven well known as reactive routing protocols create routes just when desired by the source node [8]. When a node requires a route to its destination, it initiates a route discovery process on the network. The process finishes once a route has located or all possible permutations of route were examined. Hybrid routing protocol combines both table-driven and demand-driven to transport the packets from the source to the destination.It takes the benefits of both table-driven and demand-driven routing protocols. One popular routing protocol in WMN is hybrid wireless Mesh Protocol (HWMP) [9]. It is a routing protocol for default IEEE 802.11s that consists of two components, namely: the table-driven in addition to a protocol on demand. This protocol is based on a protocol known as RM-OADV (Radio-metric ad hoc on demand Vector) routing protocol, which is the prolongation of AODV. It utilizes the same route discovery process that is used by AODV and Dynamic Source Routing (DSR). There is therefore a requirement for a routing protocol, which is as well energy efficient and scalable.

Different technique of power saving for multi-hop ad hoc wireless networks that reduces energy consumption will be proposed in this section without diminishing of the capacity or connectivity of the network. As WMNs share many common features with ad hoc networks. Thus, the approaches of energy conservation for ad hoc networks can usually be applied to WMNs. We find essentially the following approaches:

\subsection{The CDS (Connected Dominating Set)}

The CDS [10] use information of neighborhood or topology to identify all nodes which form a connected to prevail (CDS) for the network where all nodes are either a member of the CDS or immediate neighbor of at least one member selected. Nodes in the CDS are considered as the pivotal routing and remain active all the time in order to maintain global connectivity. If needed, every other node may choose to sleep. Figure 2, show the set $\{$ A, B, C, D, E, F $\}$ are the member of the CDS and remain active all the time to deliver the flow and maintain the connectivity to their neighbor. Therefore, if there is no more data transmitted by nodes $\{1-18\}$ they can choose to sleep.

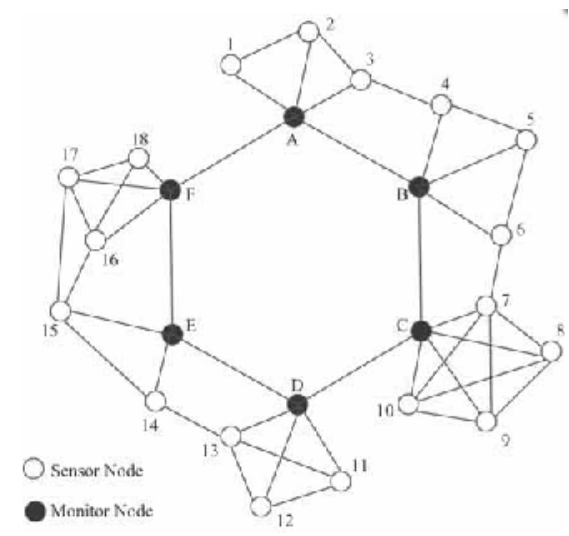

Figure 2: An example of Connected Dominating Set (CDS). 


\subsection{The SPAN}

In SPAN [11], each node in the network using Span protocol can make periodic, local decisions on the desirability to sleep or stay awake as a coordinator and take part in the transmission backbone topology. Each node decides to be a coordinator or not. The transition between the two states is made based on probabilities. Fairness is ensured by the node to a higher energy the most likely to be a coordinator. The other criterion used in selecting coordinators is the value that a node adds to the overall network connectivity. A node connecting more nodes will be more likely to be chosen as coordinator. The notion of randomness is used to prevent multiple simultaneous coordinators. Span selects adaptively "coordinators" from all nodes in network. Span coordinators keep awake permanently and carry out multi-hop packet routing into the ad hoc network, whereas the remaining nodes stay in power saving mode and periodically check whether they should wake up and become a coordinator. The principal disadvantage of this protocol is that it is inherently dependent on the node density for energy conservation.

\subsection{The GAF}

GAF [12] is another technique that uses knowledge of the geographic positions of nodes to select coordinators. The geographic positions of nodes are used to divide the complete topology in areas of fixed size (fixed geographic area). Areas are created such that any two nodes in any two adjacent zones can communicate. The size of the zone is thus dictated by the radio range of the nodes which is supposed to be fixed. Only one node in each zone must be awake and may be the coordinator. So, by exploiting the knowledge of the geographical positions GAF simplifies the selection process coordinator. The major drawback of this protocol requires that all nodes in the network recognize geographically their positions.

\subsection{EMM-DSR protocol}

In [13], authors suggested new mechanisms that allow making a trade-off between energy efficiency and the shortness of a selected path for forwarding data packets. In other word, this mechanism tries to minimize the energy consumption and, at the same time, maintain a good endto-end delay and throughput performance. Their solution consists in extending the Max-Min algorithm to support the cited trade-off. Thus, they incorporate this extension, among other options, to the existing on-demand dynamic source routing protocol (DSR), and the resultant version takes the name of EMM-DSR (Extended Max-Min DSR).

\subsection{The Minimum-energy Routing}

On minimal energy routing [14] conserves power by selecting paths through a multi-hop ad hoc network to minimize the total energy emission. This approach has been expanded by Chang and Tassiulas [15] to maximize the overall lifetime of the network by distributing energy consumption equally. In this protocol, nodes adjust their transmission power levels and choose routes for optimal performance.

\subsection{Power-Aware Routing}

In [16] a power-aware routing algorithm is presented for wireless networks with renewable energy sources. Asymptotically, the proposed algorithm proved to be optimal. No information is assumed regarding the arrival process and it is assumed that the node has full knowledge of the energy it will receive until the next renewal point by looking at previous data. The proposed routing algorithm uses a composite cost metric that includes power for transmission and 
reception, replenishment rate, and residual energy. The work also includes non-uniform energy replenishment rates and introduces a battery energy threshold scheme to decrease overhead.

\subsection{The Pulse}

The pulse protocol [17] is focused on the design of a flood event referring to a pulse that is sent regularly to a fixed pulse interval. This flood of pulses from access node infrastructure (pulse sources) and spreads across the whole network component.This rhythmic pulse fulfils two functions simultaneously. The first one was the routing mechanism mainly through periodically update every node in the route network of the closest source of pulses. Each node has followed the best path to the source pulse remembering that the node from which it received a packet of flooding with the smallest metric. The spreading of the flood formed a loop-free routing tree rooted at the source of pulses. The second is if a node needs to sent and received packets, it responds to the flooding by a reservation packet. This reservation packet is sent in the tree to the pulse source. The reservation packet has the node address of the reservation, and used to establish routes reverse to all nodes on the way between the pulse source and the sending node. A node that does not sending or transmitting a reservation packet will not have any responsibility packet until the following pulse occurs, it can set the radio in standby mode until the next pulse period begins. Disabling this node is what enables the pulse protocol to save power. Figure 3 illustrates the pulse protocol within a network, for example. Every node in the network, for example, a route to the source pulse as shown by gray arrows. Both nodes A and B are actively communicating and sending each reservation packet in the tree for the source of pulses. Packets reservation itineraries reverse configuration as shown by the black bi-directional arrows. Nodes that have forwarded a reservation stay "on" to forward data which are with the black. The remaining nodes in the network can get "off" up to the next pulse.

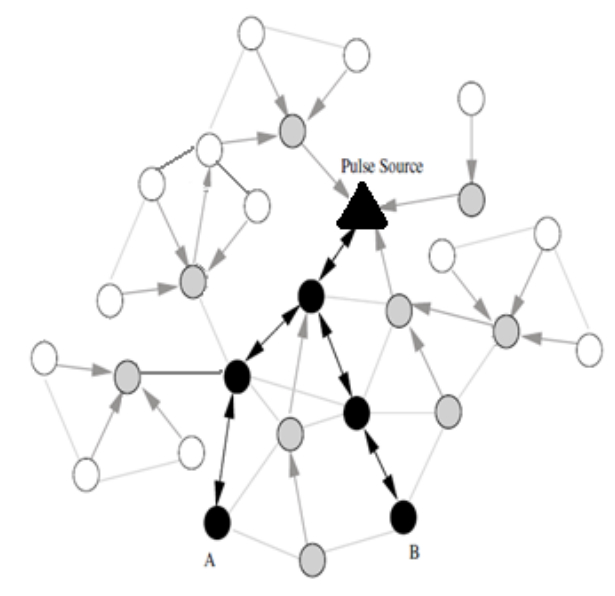

Figure 3: The Pulse protocol in an example network.

The pulse protocol [17] has significant disadvantages in the routing information that is essentially the problem of flooding packets and the problem of Overlap delay or it can result in a significant consumption of energy.

\subsection{The green-clustering}

The green-clustering algorithm [18] was developed to allow the central controller in a WLAN to make certain decisions to power on and off portions of it based on certain pre-defined criteria like deployment, location of access points, and locally derived information. Hence, this algorithm was 
designed with respect to the centralized network infrastructure and its devices. The basic idea was that if access points are close enough in the cluster, a single one would fulfil the needs of users, even those in the vicinity of other APs in the same cluster. This clustering concept was designed with respect to large organizations with high-density WLANs because access points are placed very close to each other to provide overlapping coverage and high bandwidth.

\subsection{CaDet (clustering and decision-tree-based)}

The clustering and decision-tree-based method for reducing power consumption was also approached by algorithms used on location estimation systems [19], where users are located based on the wireless signals employing a wireless device with minimum computational resources and power requirements. Using a combination of data-mining techniques and analysis of real wireless data by probabilistic location estimation and the multiple-decisions tree-based approach, some very good results can be found. These findings enable the identification of access points most used in the network, which in turn is an easy way to predict client locations. These decisions would allow a minimum of APs to be used, thus reducing the power consumption and wake-up time of the client.

\subsection{EAR (Energy Aware Routing)}

The major idea behind energy aware routing (EAR) [20] was to take energy consumption of equipment into account when doing routing and traffic-engineering decisions. In this respect, energy consumption or energy cost can be used stand-alone or in combination with other objectives and constraints like QoS or availability characteristics. EAR concept aims to switch-off completely components which certainly help to reduce the energy consumption of a network. Therefore they propose to extend the concept towards an energy profile aware routing (EPAR) and to include energy profiles into dimensioning, routing and traffic engineering decisions.

\subsection{ETR (Throughput-aware Routing)}

In [21], authors proposed a novel routing solution for 802.11 based-WMNs to provide flows with throughput guarantees while optimizing the energy consumption. The key contributions of this paper are: First, they proposed a novel way to represent the capacity region of a set of stations sharing the wireless medium, which they refer to as linearized capacity region, and compute the corresponding parameters that define this region for the case of 802.11. The linearized capacity region is devised with the aim of aiding the design of optimal and efficient networking algorithms. Second, based on the information provided by the linearized capacity region, they design a novel optimal routing algorithm for wireless mesh networks that admits as many users as possible to the network while providing them with the desired throughput guarantees. Finally, they design an extension to their routing algorithm that, in addition to providing throughput guarantees, minimizes the total energy consumed by the mesh network. This is achieved by switching off as many mesh routers as possible.

\subsection{Green Framework}

In [22], the authors developed a new framework for energy management in WMNs TDMA basic for supporting energy-efficient communications. The proposed green framework provided the WMN administrator with a parameterized objective function to choose to yield the desired tradeoff between the achieved network throughput and energy consumption. First, they proposed an Optimal Green Routing and Link Scheduling, called O-GRLS, which aims at finding the optimal trade-off. The problem was elaborated as an integer linear program (ILP). As this problem is known to be NP-hard, they then proposed a simple yet efficient algorithm based on Ant Colony, 
called Ant Colony Green Routing and Link Scheduling (AC-GRLS) to solve the formulated ILP problem. In this context, the cases of O-GRLS, a Beam Search heuristic, and Shortest Path (SP) routing strategy, are used to develop baselines to which the AC-GRLS improvements are compared. Through numerous simulations, they showed that their framework could realize significant benefits in terms of energy consumption as well as network throughput achieved, relative to the Shortest Path (SP) routing and the heuristic search Beam. Specifically, in small sized networks, for grid and random networks, the proposed energy management approaches can save, respectively, $29 \%$ and $20 \%$ of the energy cost, while achieving the same performance as SP. However, if the network consumes the same energy as SP, the achieved throughput can be enhanced by up to $30 \%$. These gains are maintained in large-sized networks. Indeed, the simulation showed that the energy saving is about $20 \%$, while the achievable throughput improvement is about $20 \%$ for both grid and random network topologies. In addition, they showed that AC-GRLS converges to the optimal solution in small sized WMNs and has low computation time in large-sized ones, which makes it a feasible and efficient solution for green WMNs and energy efficient management.

\subsection{CBRP (Cluster Based Routing Protocol)}

CBRP (Cluster Based Routing Protocol) [23] is an on-demand routing protocol, where the nodes in the network are divided into clusters. This protocol used clustering structure for routing. Each cluster has a cluster head $(\mathrm{CH})$ as coordinator. Every head of the group acts as temporarily base station across the cluster and communicating with CHs. CBRP is designed to be used in Wireless sensor network and mobile ad hoc network. The protocol divides the nodes of ad hoc network into a certain number of clusters overlapped or disjointed 2-hops in diameter in a distributed way. Each cluster selects a $\mathrm{CH}$ to retain cluster information. The node may exist in four states: Normal, Isolated $\mathrm{CH}$ and the gateway. Initially, all nodes are in the state of Isolated. Each node maintains the neighbour table where in the information about the other neighbours nodes is stored; $\mathrm{CH}$ have another table (cluster heads neighbour) where include the information about the other neighbour $\mathrm{CH}$ is stored. The protocol effectively minimizes flooding traffic during route discovery and accelerates this process as well or it may result in significant power savings.

\section{Summary}

A summary about energy saving network layer protocols, which was described in this section, will be detailed in following table.

\begin{tabular}{|l|l|l|l|}
\hline Protocol & Network & Topology & Contribution \\
\hline CDS [10] & Ad hoc & Flat & $\begin{array}{l}\text { - Use information of neighborhood to } \\
\text { determine the set of nodes which form a } \\
\text { CDS which are considers as the pivotal } \\
\text { routing and remain active all the time. }\end{array}$ \\
\hline SPAN [11] & Ad hoc & Grid & $\begin{array}{l}\text { - Local decisions on the desirability to sleep } \\
\text { or stay awake as a coordinator and take part } \\
\text { in the transmission. }\end{array}$ \\
\hline GAF [12] & Ad hoc & Grid & $\begin{array}{l}\text { - Uses knowledge of the geographic } \\
\text { positions of nodes to select coordinators. }\end{array}$ \\
\hline
\end{tabular}


International Journal of Computer Networks \& Communications (IJCNC) Vol.5, No.2, March 2013

\begin{tabular}{|c|c|c|c|}
\hline $\begin{array}{l}\text { EMM-DSR } \\
{[13]}\end{array}$ & Ad hoc & Flat & $\begin{array}{l}\text { - The selection of the shortness path for } \\
\text { forwarding data packets. }\end{array}$ \\
\hline $\begin{array}{l}\text { Minimum- } \\
\text { energy } \\
\text { Routing [14] }\end{array}$ & Ad hoc & Flat & $\begin{array}{l}\text { - Node adjusts it transmission power levels } \\
\text { and select routes to optimize performance. }\end{array}$ \\
\hline $\begin{array}{l}\text { Power-Aware } \\
\text { Routing [16] }\end{array}$ & Ad hoc & Flat & $\begin{array}{l}\text { - Routing algorithm uses a composite cost } \\
\text { metric that includes power for transmission } \\
\text { and reception, replenishment rate, and } \\
\text { residual energy. }\end{array}$ \\
\hline Pulse [17] & $\begin{array}{l}\text { multi-hop } \\
\text { wireless } \\
\text { infrastructure }\end{array}$ & Tree & $\begin{array}{l}\text { Flood known as a pulse, which is } \\
\text { periodically sent at a fixed pulse interval to } \\
\text { monitor the right way for the pulse source. }\end{array}$ \\
\hline $\begin{array}{l}\text { Green- } \\
\text { clustering [18] }\end{array}$ & $\begin{array}{l}\text { WLAN } \\
\text { infrastructure }\end{array}$ & Clustered & $\begin{array}{l}\text { - Central control to make certain decisions } \\
\text { to power on and off portions of it based on } \\
\text { certain pre-defined criteria. }\end{array}$ \\
\hline CaDet [19] & $\begin{array}{l}\text { WLAN } \\
\text { infrastructure }\end{array}$ & Clustered & - Clustering and decision-tree-based. \\
\hline EAR [20] & All networks & Flat & $\begin{array}{l}\text { - Take energy consumption of equipment } \\
\text { into account when doing routing and } \\
\text { traffic-engineering decisions. }\end{array}$ \\
\hline ETR [21] & $\begin{array}{l}\text { Wireless Mesh } \\
\text { Networks }\end{array}$ & Flat & $\begin{array}{l}\text { - Routing algorithm that uses as few nodes } \\
\text { as possible, this allows switching off the } \\
\text { unused nodes. }\end{array}$ \\
\hline $\begin{array}{l}\text { Green } \\
\text { Framework } \\
{[22]}\end{array}$ & $\begin{array}{l}\text { Wireless Mesh } \\
\text { Networks }\end{array}$ & Flat & $\begin{array}{l}\text { - Optimal Green Routing and Link } \\
\text { Scheduling. } \\
\text { - Ant Colony Green Routing and Link } \\
\text { Scheduling. }\end{array}$ \\
\hline CBRP [23] & $\begin{array}{l}\text { Wireless Mesh } \\
\text { Networks }\end{array}$ & Clustered & $\begin{array}{l}\text { Minimizes the flooding traffic during route } \\
\text { discovery. }\end{array}$ \\
\hline
\end{tabular}

In terms of routing, we think the approach of clustering is the most interesting to conserve energy consumption. The selection of the cluster head in each cluster on the basis of energy level in wireless mesh network can reduce the rate of energy consumption by scheduling activities in the cluster of mesh users but not for mesh routers. 
The Pulse protocol is designed for multi-hop wireless infrastructure access and an extensive set of simulations had demonstrated that this protocol is effective at both routing and conserving energy.

\section{ENERGY CONSERVATION IN DATA LINK LAYER}

In the previous section, we showed approaches that conserve energy in Network Layer. We will now discuss some of the key issues concerning the implemented energy conservation in MAC layer sub-layer of the data link that supplies a fair mechanism for shared access medium amongst other nodes. The MAC plays a key role in the maximization of node's energy efficiency. We first briefly review power saving in conventional IEEE 802.11 and discussion of recent work that has started to address them.

\subsection{PSM (Power Saving Mode) IEEE 802.11 Protocol}

The 802.11 standard defines a power saving mode (PSM) [24] to reduce the energy consumption of mobile devices. The purpose of PSM is to leave 802.11 wireless interface of a mobile host in the active mode just for the time needed to share data, and transform it into the sleep mode once it becomes idle. PSM defining two modes of the power management has changed mobile device can operate in one of them: the active mode and power saving mode (PSM). In active mode, a mobile device is completely powered and is able to exchange frames at any time. In the power saving Mode, a mobile device is authorized to be in one of two different power states, whether in awake state or doze state. Access points (AP) in wireless network Stock observe every mobile device. A mobile device must first inform its access point (AP) about changing its power management mode using Power Management bits within the Frame Control field of the frame used as a power saving request. During the association procedure, a mobile device is informing the access point AP in its listening interval which is made to indicate a period of time chosen by a mobile device in the PSM can choose to sleep. Its aim is to reduce energy consumption in conventional IEEE 802.11 standard.

A mobile device running PSM can go to sleep which incurs power consumption of only around $50 \mathrm{~mW}$. However, when a PSM device is sleeping, it cannot transmit or receive any packets; hence, PSM clients conserve energy at the cost of larger packet delivery delay.

\subsection{APSD (Automatic Power Save Delivery)}

Various enhancements to the above scheme have been included in some of the follow-on standards. For example, IEEE 802.11e defines Automatic Power Save Delivery (APSD) [25]. This includes both contention-based operation (referred to as EDCA) and a polling-based option (called HCCA). In the latter case, the access point (AP) functions as a hybrid coordinator (HC), and defines periodic service intervals that allow the synchronous delivery of traffic using Scheduled Automatic Power Save Delivery (S-APSD). In the former case the unscheduled APSD (U-APSD) mechanism permits the station to initiate communication activity by transmitting trigger frames on the uplink in EDCA contention mode. These mechanisms provide for improved flexibility and power saving compared to the original procedures.

\subsection{PSMP (Power Save Multi-Poll)}

IEEE 802.11n has introduced further enhancements to the U-APSD and S-APSD protocols, referred to as power save multi-poll (PSMP) [26]. As in its predecessors, there are scheduled (i.e., S-PSMP) and unscheduled (i.e., U-PSMP) versions. S-PSMP provides tighter control over the AP/station timeline by having the AP define a PSMP sequence that includes scheduled times for 
International Journal of Computer Networks \& Communications (IJCNC) Vol.5, No.2, March 2013

downlink and uplink transmissions. The ability to do this allows (non-AP) stations to remain in Doze mode during the times when other stations are scheduled to be using the channel and reduces AP/station interaction overheads. U-PSMP is similar to U-APSD in that it supports both triggered and delivery enabled modes.

\subsection{NAV (Network Allocation Vector) and NAM (Network Allocation Map)}

The access point (AP) uses network allocation vector blocking to prevent channel access to the AP when it is in Doze mode [27]. In conventional IEEE 802.11, a NAV is used at each station to implement a virtual carrier sense mechanism and to block stations from transmitting in cases where the channel has been reserved for some other purpose.

The mechanism is widespread IEEE 802.11 NAV and energy savings AP comprises a Network Allocation Map (NAM) in its beacon broadcasts [28]. NAM specifies times within the super frame when the AP is unavailable, and during these times, the AP is supposed to be inactive and power conservation.

\subsection{PAMAS (Power-aware Multi Access Protocol with Signalling)}

The PAMAS [29] protocol for medium access protocol suggested turning off the radio when it is overhearing a packet not addressed to it. It is a combination of original MAC protocol, and using a separate channel for a busy signal. Using the busy signal, the terminals are able to determine when and how long they should turn off their radio interfaces. In this protocol, if a node has no packet to transmit, then it should turn off its radio interface if one of its neighboring nodes begins transmitting. Similarly, if at least one neighboring node transmits and another receives, the node should also turn off power because it cannot transmit or receive packets (even if its transmission queue is not empty). This approach uses a separate channel for a busy signal. Each node listens to channel to see when it becomes free to transmit (idle-listening). So it leads to important energy consumption.

\subsection{SOFA (A Sleep-Optimal Fair-Attention scheduler)}

In SOFA [30] authors proposed a downlink traffic scheduler on the AP of a WLAN, called SOFA, which help its PSM clients to save energy by allowing them to sleep more, hence to increase battery lives. If a client has buffered packets at the AP in a beacon period, and that client decides to receive it, it has to remain awake from the beginning of the beacon period till the last packet scheduled for it in the beacon period is delivered. Therefore a large portion of energy wastage (for the client) comes from the AP transmitting other clients' a packets before it finishes transmitting the client's last packet to it. SOFA manages to reduce such energy wastage and maximizes the total sleep time of all clients. SOFA favors clients with smaller attention requests by allowing them to spend less energy to get one unit of attention, while still helping other clients with larger attention requests to sleep more compared with other popular scheduling policies like round-robin and FCFS.

\subsection{LEACH (Low-Energy Adaptive clustering Hierarchy)}

LEACH is scheduled MAC protocol with clustered topology [31]. It is considered the first hierarchical routing protocol. It is also one of the hierarchical routing algorithms for WSN popular, it combines the efficiency of energy consumption and quality of media access, and based on the division into clusters, view to enable the use of the concept of data aggregation for better performance in terms of lifetime. 
The communication architecture of LEACH is, similarly to the cellular network to form cells based on the signal amplitude, and using the cell headers to node routers as well. These cells are known as Cluster-heads $(\mathrm{CH})$. Selection of the $\mathrm{CH}$ is randomly done according to a specific algorithm choice based on a likelihood function that takes into account different factors particularly the disposable energy of nodes. As Figure 4 shows, the nodes are responsible for collecting data, send them to their $\mathrm{CH}$ aggregate and transmit them, in turn, results aggregation node well as a communication unicast (one hop). CHs are responsible for carrying out the functions most expensive energy, namely communication with the node sinks which is assumed far, and all data processing (aggregation, fusion and data) to reduce the amount the transmitted data. This device saves energy since the transmissions are provided solely by $\mathrm{CH}$ rather than all nodes in the network. Therefore, LEACH achieves a significant reduction in energy dissipation.

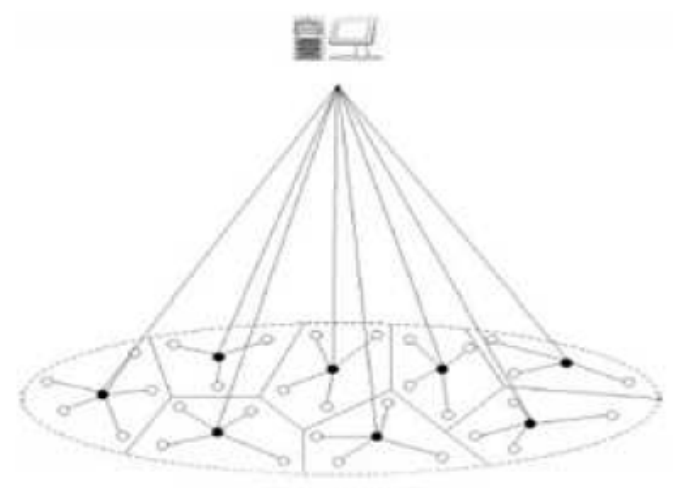

Figure 4: The architecture of Communications for LEACH protocol.

\subsection{S-MAC}

S-MAC [32] protocol is energy efficient MAC protocol toward wireless sensor networks for reducing energy consumption, while sustaining good scalability and collision avoidance. S-MAC takes an effective mechanism for solving the problems of losing energy, which is periodic listening and sleeping. When a node is idle, it is more likely to be asleep rather than continuously listening on the channel. S-MAC reduces the listen time by leaving the node goes into sleep mode periodically. The main aim of S-MAC is minimizing energy consumption, including three major elements: the situation wake, periodic sleep and listen. This protocol aim to avoid collision and overhearing that implies that in this protocol, nodes go to sleep after hearing RTS or CTS packet and the duration field in each transmitted packet shows how long the remaining transmission will be and the communication between the sender that the message passing is shown in Figure 5.

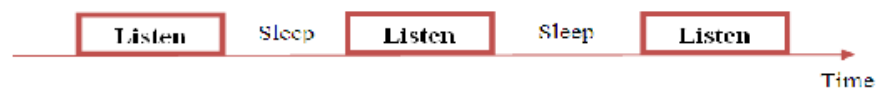

Figure 5: Periodic Listen and Sleep

\subsection{Virtualization of NICs}

In [33], one of the possible ways to save energy is to shutdown certain access points that serve a specific field of interest, if the number of stations served are decreased within a few hours peak (eg at night, weekends, holidays). This might lead to the fact that some locations in the zone concerned are not covered, and the stations situated in these areas will lack the connections. They can obtain connectivity using extension of network coverage / capacity relay. In addition to the 
energy saved by shutting down some APs, NIC virtualization plays a role in reducing energy consumption.

A scenario represented in Figure 6 and Figure 7, in which specific area is covered by a number of APs. This number is reduced by shutting down some of them if the number of the served stations is decreased in off-peak hours. In Figure 6, the whole area is served by six powered-on APs. Every circle represents an AP's coverage area. The symbols inside these cells represent stations. Stations with the same shape and colour are served by an AP. While in Fig. 3, only two APs are powered-on to reduce the consumed energy.

The number of APs is only reduced since the number of currently jointed stations is smaller than those in Figure 6. It's clearly shown in Figure 7 that the remaining powered-on APs do not cover the whole area. Thus, stations located outside (surrounded by dotted squares. These are equipped with virtualized NICs so that a station uses one of its virtual interfaces to create a network with other stations while keeping its connection to the serving AP using another virtual interface) the coverage range of the APs can get access to the Internet via stations (work as relays) connected to any served AP.

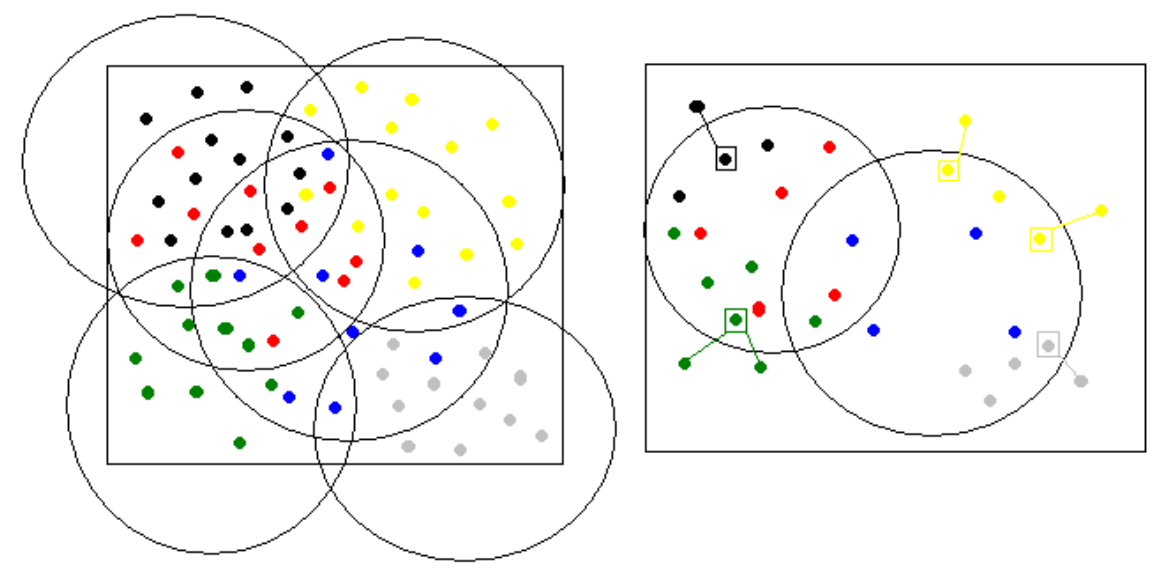

Figure 6: Stations served with 6 powered-on APs in a specific covered area.

Figure 7: Stations served with 2 powered-on APs in a specific covered area, some stations are equipped with virtual NICs to act also as APs and serve the stations existing in uncovered locations.

\subsection{PEM (Power-Efficient MAC Protocol)}

In [34] authors developed an efficient MAC protocol, PEM, to enhance the network utilization and reduce energy consumption. In PEM, stations can estimate their distances from the transmitter and obtain the interference relations among transmission pairs through three way-way handshaking (ATIM/ATIM-ACK/IIM). According to the interference relation, a scheduling algorithm is proposed for stations to schedule their transmissions. The scheduling algorithm can select interference-free transmission pairs as many as possible to increase the spatial reuse. Thereafter, a station intending to transmit can adjust its transmission power to transmit at its scheduled time In PEM; every station knows when should wake up and when they can enter into sleepy state. Thus, stations can save much energy. The network bandwidth may be used efficiently as well. Simulation results show that PEM outperforms than existing protocols, such as DCF, DPSM, and DCS. PEM not only reduces power consumption, but also increases the network throughput. 
International Journal of Computer Networks \& Communications (IJCNC) Vol.5, No.2, March 2013

\subsection{MT-MAC (Multi-hop TDMA Energy-efficient Sleeping MAC Protocol)}

In order to improve the performance of energy efficiency, throughput and delay in WMSN, the authors [35] designed a Multi-hop TDMA Energy-efficient Sleeping MAC (MT-MAC) protocol. The main idea of MT-MAC is to divide one frame into many slots for sensor nodes in WMSN to forward data packets, with TDMA scheduling method. At first, each node tries to get routing information from its routing layer while constructing the neighbor list through flooding algorithm. Then, after collecting the necessary information, each node transfers both the routing information and neighbor information to the mesh router. And the mesh router computes the slot allocation table called the schedule. At last, the mesh router broadcasts both the schedule and its own Synchronous clock. So every node not only keeps synchronized with the mesh router, but also decides when to forward data according to the schedule. As collision and hidden terminal problems are considered in the scheduling algorithm, so the probability of collision in the network will be significantly reduced, and the network throughput will be improved. As the simulation result showed, compared with SMAC protocol, MT-MAC not only saves $25 \%$ energy consumption of the network, but also decreases $45 \%$ latency of the whole network.

\section{Summary}

The discussed MAC protocols are summarized and classified in the table below.

\begin{tabular}{|l|l|l|l|l|}
\hline Protocol & Network & Topology & $\begin{array}{l}\text { Channel/ } \\
\text { Synchronization }\end{array}$ & Contribution \\
\hline PSM [24] & 802.11 & Flat & $1 /$ Yes & Active/sleep period \\
\hline APSD [25] & $802.11 \mathrm{e}$ & Flat & $1 /$ Yes & $\begin{array}{l}\text { Active/sleep } \\
\text { schedules }\end{array}$ \\
\hline PSMP [26] & $802.11 \mathrm{n}$ & Flat & $2 /$ Yes & $\begin{array}{l}\text { Active/sleep with } \\
\text { schedules } \\
\text { reducing overheads }\end{array}$ \\
\hline NAV [27] & 802.11 & Flat & $1 /$ Yes & $\begin{array}{l}\text { Active/sleep with } \\
\text { blockage } \\
\text { transmission when } \\
\text { channel is reserved }\end{array}$ \\
\hline MAN [28] & 802.11 & Flat & $1 /$ Yes & $\begin{array}{l}\text { Map of active/sleep } \\
\text { period }\end{array}$ \\
\hline PAMAS [29] & Ad hoc & Flat & $2 /$ No & $\begin{array}{l}\text { Wake up radio } \\
\text { scheme }\end{array}$ \\
\hline SOFA [30] & 802.11 & Flat & $1 /$ NO & $\begin{array}{l}\text { A round-robin } \\
\text { scheduling }\end{array}$ \\
\hline
\end{tabular}


International Journal of Computer Networks \& Communications (IJCNC) Vol.5, No.2, March 2013

\begin{tabular}{|l|l|l|l|l|}
\hline & & & & sleep/wakeup \\
\hline LEACH [31] & 802.15 .4 & Clustered & $1 /$ Yes & $\begin{array}{l}\text { Low energy } \\
\text { clustering }\end{array}$ \\
\hline S-MAC [32] & 802.15 .4 & Flat & $1 /$ Yes & $\begin{array}{l}\text { Active/sleep } \\
\text { schedules }\end{array}$ \\
\hline $\begin{array}{l}\text { Virtualization } \\
\text { of NICs [33] }\end{array}$ & 802.11 & Flat & $1 /$ Yes & $\begin{array}{l}\text { Virtualization of } \\
\text { network interface } \\
\text { cards ard and }\end{array}$ \\
\hline PEM [34] & 802.11 & Flat & $1 /$ No & $\begin{array}{l}\text { Power control aning } \\
\text { power } \\
\text { mechanisms }\end{array}$ \\
\hline MT-MAC [35] & WMSN & Clustered & $1 /$ Yes & $\begin{array}{l}\text { TDMA Energy- } \\
\text { efficient Sleeping } \\
\text { MAC }\end{array}$ \\
\hline
\end{tabular}

The Data Link Layer is very important to ensure energy conservation for Wireless Mesh Networks. Many researchers are focused on specific approaches as we have seen previously. The most of researches used power save mode mechanism based on 802.11 standard or the TDMA scheduling method to improve and better energy saving.

\section{ENERgy CONSERVATION IN PHySiCAL LAYER}

Physical Layer (PHY) implements network communication equipment that sends and receives messages from a bit or symbol at a time. The researches that involve in the Physical Layer of WMNs are pointed out in this Section.

\subsection{The LM-SPT (Local Minimum Shortest Path-Tree)}

This work [36] addressed the challenge of power efficiency by presenting a localized distributed power-efficient topology control algorithm for application of WMNs in rural areas. The main idea in this approach was to construct an overlay graph topology of the WMN with such desirable features as reduced physical node degree, increased throughput, increased network lifetime and maintenance of connectivity by varying the transmission power at each node. The main contribution of this work was the ability of the algorithm to balance energy efficiency and throughput in WMNs and without loss of connectivity. The concept of this approach was based on information of the local neighborhood that is confined to one hop from the logical visible neighborhood for calculating the minimum power transmission. In this approach Aron et al. [36] proposed to calculate minimum transmission power and require only an optimal value to maintain an optimal connectivity and decrease the interference and collision which save energy and achieve a good throughput. 


\subsection{CNN (Critical Number of Neighbors)}

Santi [37] used CNN refers to the minimum number of neighbors that should be maintained by each node in the network to be asymptotically connected. This approach is adopted to maintain connectivity, since only the knowing the size of the network is needed to determine CNN. This information can be easily obtained from a proactive routing protocol like OLSR. CNN can also result in heterogeneous transceiver power outputs, which could maximize energy savings and gains interference. CNN is also less affected by the distribution and location of network nodes and there is no need to assume a GPS enabled router. It is also increases progressively with the size of the network and is capable of tolerating delays in the propagation of updates to topology and size of the network (if a proactive routing protocol is used). Thus, maintaining connectivity via CNN will help reduce human intervention. A similar idea to Aron et al. [36] approach, Santi [37] proposed to calculate minimum number of neighbors that should be maintained to be connected to conserve energy.

\subsection{Minimum-energy topology}

Aron et al. [38] considered the problem of topology control in a hybrid WMN of heterogeneous and presented a localized distributed topology control which pointed to calculate the optimal transmission power to maintain connectivity and reduce the transmission power to cover only nearest neighbors with saving energy and extending the lifetimes of the networks. In this approach, the main objective was to generate a minimum-energy topology $G$ graph. Taking an arbitrary node $u \in \mathrm{V}$ in the network $\mathrm{G}$, a three phased topology control algorithm those run in each node. First, the node $u$ broadcasts a "Hi" message using its full power, $P^{\max }{ }_{u}$. The nodes that receive the "Hi" message form the set of accessible neighborhood of node $u$. The "Hi" message contains the id of $\mathrm{u}$, the location information of $u,(\mathrm{x}(\mathrm{u}), \mathrm{y}(\mathrm{u}))$ and the value of the $P^{\max }{ }_{u}$. After there exist a weighted directed graph topology G, $u \in \mathrm{V}$. Node $u$ has knowledge of the edge weights and path weights, where path weight of a directed path. Finally, the node $u$ determines its own transmission power and the power on the accessible edges of all the nodes in the accessible neighborhood. Node $u$ takes as its power, the largest one-hop edge weight among the edges obtained in the minimum-energy local topology view $\mathrm{G}$, after node $\mathrm{u}$ adopt its minimum-energy level; it propagates this minimum power value to the other neighbors in the accessible neighborhood with the current Transmission power. Aron et al. [38] developed a minimumenergy distributed topology control that ensures a reduction in the amount of energy consumed per node during transmissions and without loss of connectivity with increasing in the network lifetime.

\subsection{PlainTC}

Mudali et al. [39] investigated the feasibility of power control in a popular WMN backbone device and design and assess an autonomous, lightweight system known as TC PlainTC. Two main approaches may be used in this context, the maintenance of the Critical Transmission Range (CTR) or the Critical Neighbor Number (CNN). In this work, Mudali et al. introduced a scheme for TC backbone WMN including routers WRT54GL Linksys available on the market (which are popular devices backbone $\mathrm{WMN}$ ). The proposed system is adapted to maintain network connectivity based on data gathered by a proactive routing protocol. Three kinds of information can be collected and used as the basis of a TC system: information location, direction information and neighbour information. Linksys WRT54GL device is free of native GPS or the ability of determining the relative direction of incoming and outgoing transmissions. The device has the capacity to collect low-quality and neighbor based information by inspecting the route table built by proactive routing protocol is used. Determining the degree of logical node is easier since the 
number of HELLO messages received from unique sources may be determined whether a reactive routing protocol is used. Whether a proactive routing protocol is used, then the route table can be examined to the number of one-hop (or n-hop) neighbors. The approach proposed by Mudali et al. [39], is designed for a specific device (Linksys WRT54GL) and it contains a GPS to define the direction. Unfortunately, generally the devices are not specific and they are not equipped with a GPS generally.

\subsection{Virtual WLAN}

Coskun et al. [40], proposed the switching scheme which aims to powering on the minimum number of devices or the combination of devices that consume the least energy that can jointly provide full coverage and enough capacity. This approach corresponds to having a minimum set of devices that provide coverage and an additional set of devices that are powered on to provide additional capacity when needed. By consolidating hardware, some hardware can be put in lowpower mode and energy consumption can be reduced, saving the difference in energy consumption per low-power node when compared to an active node and adding the amount of energy consumed by hosting more networking processes just on fewer network nodes. Depending on the type of device, different amounts can be saved. Using virtual 802.11 interfaces to connect to multiple networks simultaneously, instead of using multiple network interface cards, enables savings in energy costs, minimizes the physical space, and provides the capability to build large and small wireless mesh networks.

\subsection{Sleeping and Rate-Adaptation}

Nedevschi et al. [41] have presented the design and assessment of two management systems which reduce power consumption of the networks. The first one is to put the network interfaces to sleep for brief periods. To make this successful they introduce various small amounts of buffer, although 802.11 AP (access point) to sleep for clients who reassembles the packets into smaller bursts and thus creates gaps long enough to sleep profitable. Preoccupations are that the buffering will add too much delay over the network and the bursts will only aggravate the loss. Algorithms hold their routers and switches for sleeping in a manner that ensures the buffering delay penalty is paid once (not per link) and burst clear routers to not amplify the significant loss. The outcome is a new mechanism which differs from 802.11 schemes that all components of network are able to sleep when it is not being used further added delay is bounded. The second approach adapts the rate of individual links based on the utilization and queuing delay of the link.

\subsection{Connectivity strategies}

Mudali et al. proposed [42]; various connectivity strategies based on the Critical Number of Neighbors approach are evaluated via simulation to determine the relationship between transceiver power savings and the network lifetime. The evaluation indicates that the selected connectivity strategies are able to produce cumulative transceiver power savings. The extent of the power savings produced by individual backbone nodes is largely dependent upon the location of the node relative to the (imaginary) center of the backbone network. The evaluation also suggests that cumulative transceiver power savings do not automatically translate into corresponding extensions of network lifetime.

\subsection{Greening of the Internet}

Gupta and Singh [42] identified the problem of excessive energy consumption in the Internet and proposed sleep as the approach to conserve energy. They thought that sleeping was a great way to maximizing the energy conservation of users. However, in order to implement the algorithms for 
sleeping on one hand needs a redesign of materials network equipment, that has been made to enable sleep compatible software. Other routing protocols have been amended to adapt energy consumption and permit its load via aggregation and sleep. Thirdly, for additional options for selecting the route aggregation and sleep, the topology of the Internet has been modified as well, and finally, the investigation of the sleep impact on the protocols such as TCP with an eye on the modification of the protocol so as to adapt to the presence of sleeping nodes. It emerged that sleep was in fact a feasible strategy, but it will make certain changes to the specifications of the current protocol. In addition, in order to maximize the amount of energy savings, they note that certain changes in the architecture of the Internet may be required (including adding links to allow packet aggregation along the roads less).

\subsection{Power Control and Rate Adaptation}

In [44], authors study the scheduling optimization problem in wireless MESH networks assuming a time division multiple access (TDMA) system, a dynamic power control capable of varying emitted power slot per slot, and a rate adaptation mechanism that sets transmission rates according to the SINR. Constraints of quality traffic are expressed in terms of minimum bandwidth. From the period determined by the TDMA system is fixed, the need for bandwidth may be converted into the number of information units (packets) to be transmitted on each link per frame. Moreover, according to a discrete set of possible transmission rates, the number of packets, which may be transmitted by time interval, depends on the SINR at the receivers. To further ideas about the features of the issue and the effect of various control mechanisms, they considered three different versions of the problem with increasing complexity. In the first one they assume fixed power and rate, in the second one variable power and fixed rate, and finally in the third one variable power and rate. Given a number of available slots, the goal is to provide an assignment of time slots to links such that bandwidth constraints are satisfied and the number of available time slots is not exceeded.

\subsection{Energy savings in a time-variable context}

In [7], the authors suggested a new approach for the dynamic energy management of WMNs to minimize the energy in a time-varying dynamically by choosing a subset of base stations to switch the mesh examination matters cover the service zone, traffic routing, as well as capacity limitations both on the segment access and wireless backhaul. To reach this objective, they provided an optimizing framework on the basis of mathematical programming that takes into account the traffic requirements for a set of time intervals and handles energy consumption of the network for the purpose to make it proportional to the load. Contributions provided by the authors are as follows: First, they took into consideration the access segment and the wireless link of wireless access networks. Second, they combined all the matter of wireless coverage for the access segment and delivery by the transport network, and optimized jointly. Third, the authors include explicitly traffic variations on a set time interval and demonstrate how it is possible to have the energy after these changes. Finally, they provide a rigorous mathematical modeling of the problem of minimizing power based on Mixed Integer Linear Programming (MILP) and resolve it optimally.

\section{Summary}

Topology control algorithms have largely been proven to be one way of achieving energy efficiency in MWNs. Many contributions have been adapted to study the problems of power control in energy constrained conventional IEEE 802.11 wireless network, little attention was drawn to the power control issues in WMNs. Control of topology and control of power transmission are the most widely used, simple but very effective for saving energy. 
International Journal of Computer Networks \& Communications (IJCNC) Vol.5, No.2, March 2013

\section{Conclusions}

Various existing energy conservation methods proposed by different studies but the energy is still a challenging issue for wireless mesh network because of its significant environmental footprint. In this paper, we have summarized certain research results which have been reported in the literature on methodologies for energy conservation in wireless mesh networks. Even though lot of these power saving techniques seem promising, there are still a lot of challenges that must be addressed. Therefore, other research is required to process these kinds of situations. On the basis of these studies have been previously conducted by different researchers we reach at the obvious conclusion that if we want to conserve energy in $\mathrm{WMN}$, the most effective way is to combine the most effective solutions in the three lower layers but it still a challenge because a cross-layer difficulties.

\section{REFERENCES}

[1] S.Mamechaoui, F.Didi and G.Pujolle, (2012) "Energy Saving For Wireless Mesh Network" MESH 2012 : IARIA, 2012. ISBN: 978-1-61208-210-3.

[2] S.Mamechaoui, F.Didi and G.Pujolle, (2012) "Power Conservation for Wireless Mesh Network" 2012 IEEE 24th International Conference on Microelectronics (ICM).

[3] I.F. Akyildiz and X. Wang, (2005) "A survey on wireless mesh networks". IEEE communications Magazine 43(9), s23-s30.

[4] I.F. Akyildiz, X. Wang and W. Wang, (2005) "Wireless mesh networks: a survey". Computer Networks Journal (Elsevier) 47(4), pp 445-487.

[5] The Wi-Fi Alliance. http://www.wi-fi.org/.

[6] The WiMAX Forum. http://www.wimaxforum.org/home.

[7] A. Capone, F.Malandra, B. Sansò, (2012) "Energy Savings in Wireless Mesh Networks in a TimeVariable Context". Mobile Networks and Applications, Volume 17, Issue 2, pp 298-311.

[8] S. Giuannoulis, C. Antonopoulos, E. Topalis, and S. Koubias, (2005) "ZRP versus DSR and TORA: A comprehensive survey on ZRP performance".10th IEEE Conference on Emerging Technology Automation, 8 pp. - 1024 .

[9] Michael Bahr; (2006) "Proposed routing for IEEE 802.11s WLAN mesh networks". WICON '06 Proceedings of the 2nd annual international workshop on Wireless internet, Article No.5.

[10] M. Cardei, M.X. Cheng, X. Cheng, Du D.-Z., (2002) "Connected Domination in Ad Hoc Wireless Networks", Proceedings of the Sixth International Conference on Computer Science and Informatics (CSI).

[11] B.Chen, K. Jamieson, H. Balakrishnan and R. Morris, (2002) "Span: An Energy-Efficient Coordination Algorithm for Topology Maintenance in Ad Hoc Wireless Networks", Kluwer Academic Publishers. Manufactured in The Netherlands, 2002.

[12] Y. Xu, J. Heidemann and D. Estrin, (2001), “ Geography-informed energy conservation for ad hoc routing”, Proceedings of the Seventh Annual ACM/IEEE International Conference on Mobile Computing and Networking (MobiCom), 2001.

[13] B. Bouyedou , M.FEHAM, F. Didi, H. Labiod,(2009) "Improvement of DSR Performances in Mobile Ad hoc Networks with Trade-off between Energy Efficiency and Path Shortness", IEEE International workshop on ITS for an Ubiquitous Roads UBIROADS 2009, Hammamet, Tunisia, June 2009.

[14] T. Sheppard, "A channel access scheme for large dense packet radio networks", Proceedings of the ACM SIGCOMM, 1996.

[15] J. Chang and L. Tassiulas, "Energy conserving routing in wireless ad hoc Networks", Proceedings of the Fifth Annual ACM/IEEE International Conference on Mobile Computing and Network (MobiCom), Dallas,TX (August 1998). 
International Journal of Computer Networks \& Communications (IJCNC) Vol.5, No.2, March 2013

[16] L. Lin, N.B. Shroff, and R. Srikant. "Asymptotically Optimal Power- Aware Routing for Multihop Wireless Networks with Renewable Energy Sources”. INFOCOM 2005.

[17] B. Awerbuch, D. Holmer, and H. Rubens, "The Pulse Protocol: Energy Efficient Infrastructure Access", IEEE INFOCOM, 2004.

[18] Iannaccone, Jardosh, Papagiannaki and Vinnakota, (2007) "Towards an Energy-Star WLAN infrastructure”, Mobile Computing Systems and Applications, Hotmobile., Eighth IEEE Workshop., pp. 85-90, 2007.

[19] Chen, Chin, Yang and Yin, "Power-Efficient Access-Point Selection for Indoor Location Estimation", IEEE Transactions and Data Engineering, Vol. 18, No.7, pp. 877-888, DOI:

[20] J. Restrepo, C. Gruber, and C. Machuca, (2009), "Energy profile aware routing," in Communications Workshops, 2009. IEEE International Conference on, pp. $1-5$.

[21] A. Oliva, A. Banchs and P. Serrano, (2012), "Throughput and energy-aware routing for 802.11 based mesh networks”, Comput. Commun. http://dx.doi.org/10.1016/j.comcom.2012.04.004.

[22] Amokrane Ahmed, Langar Rami, Boutaba Raouf and Pujolle Guy; (2012), "A Green Framework for Energy Efficient Management in TDMA-based Wireless Mesh Networks”; IEEE/ACM CNSM 2012 , Las Vegas, USA.

[23] C. Bemmoussat, F. Didi, M. Feham, (2012) "EFFICIENT ROUTING PROTOCOL TO SUPPORT QOS IN WIRELESS MESH NETWORK”, International Journal of Wireless \& Mobile Networks (IJWMN) Vol. 4, No. 5, October 2012.

[24] IEEE 802.11, Wireless LAN Medium Access Control (MAC) and Physical Layer (PHY) Specifications, 1999.

[25] IEEE Stds. Dept.. "Part 11: Wireless Medium Access Control (MAC) and Physical Layer (PHY) Specifications: Medium Access Control (MAC) Quality of Service (QoS) Enhancements”, IEEE Press, 2005.

[26] IEEE Stds. Dept., "Part 11: Wireless LAN Medium Access Control (MAC) and Physical Layer (PHY) specifications: Amendment 4: Enhancements for Higher Throughput”, IEEE P802.11n/D3.00. IEEE Press, 2007.

[27] F. Zhang et al., "Power Saving Access Points for IEEE 802.11 Wireless Network Infrastructure", IEEE Wireless Commun. and Networking Conf. 2004, March 2004.

[28] Y. Li, T. D. Todd, and D. Zhao. "Access Point Power Saving in Solar/Battery Powered IEEE 802.11 ESS Mesh Networks”, 2nd Int'l. Conf. Quality of Service in Heterogeneous Wired/Wireless Networks, Augst 2005.

[29] S.Singh and C.S.Raghavendra, "Power aware multi-access protocol with signaling for ad hoc networks", ACM Computer Communication Review, Vol. 28 No. 3, pp. 5-26, July 1998.

[30] Z. Zeng, Y. Gao, and P. R. Kumar, SOFA: A Sleep-Optimal Fair-Attention scheduler for the PowerSaving Mode of WLANs, 31st International Conference on Distributed Computing Systems, 2011 IEEE.

[31] W.R. Heinzelman, A. Chandrakasan, and H. Balakrishnan. "Energy-Efficient Communication Protocol for Wireless Micro Sensor Networks". In IEEE Proceedings of the Hawaii international Conference on System Sciences (HICSS '00), 2002.

[32] W. Ye, J. Heidemann, D. Estrin, “An Energy- Efficient MAC Protocol for Wireless Sensor Networks", IEEE INFOCOM 2002.

[33] Y. Al-Hazmi and Hermann, "Virtualization of 802.11 Interfaces for Wireless Mesh Networks", Wireless On-Demand Network Systems and Services (WONS), 2011 Eighth International Conference on 26-28 Jan 2011.

[34] K. Shih, C. Chang, C. Min Chou, S. Chen, "A Power Saving MAC Protocol by Increasing Spatial Reuse for IEEE 802.11 Ad Hoc WLANs”, AINA '05 Proceedings of the 19th International Conference on Advanced Information Networking and Applications - Volume 1 Pages 420-425

[35] Q. Fan, J. Fan1, J. Li, and Xiaofang Wang; (2012), "A Multi-hop Energy-efficient Sleeping MAC Protocol based on TDMA Scheduling for Wireless Mesh Sensor Networks", JOURNAL OF NETWORKS, VOL. 7, NO. 9, SEPTEMBER 2012

[36] F.O. Aron, A. Kurien and Y. Hamam, “ Topology Control Algorithm for Effective Power Efficiency and Throughput for Wireless Mesh Networks", Third International Conf. on Broadband Communications, Information Technology \& Biomedical Applications, 2008. 
International Journal of Computer Networks \& Communications (IJCNC) Vol.5, No.2, March 2013

[37] P.Santi, “Topology Control in Wireless Ad Hoc and Sensor Networks", Wiley: Chichester, 2005.

[38] F. O. Aron, T. O. Olwal, A. Kurien, and M. O. Odhiambo, “A Distributed Topology Control Algorithm to Conserve Energy in Heterogeneous Wireless Mesh Networks", World Academy of Science, Engineering and Technology 40, 2008.

[39] P. Mudali, T.C. Nyandeni, N. Ntlatlapay, and M.O. Adigun, "Design and Implementation of a Topology Control Scheme for Wireless Mesh Networks", IEEE AFRICON 2009.

[40] H. Coskun, I. Schieferdecker and Y. Al-Hazmi, "Virtual WLAN: Going beyond Virtual Access Points", ECEASST, vol. 17, March 2009.

[41] S. Nedevschi, L. Popa, G. Iannaccone, "Reducing Network Energy Consumption via Sleeping and Rate-Adaptation", Proceedings of the 5th USENIX Symposium on Networked Systems Design and Implementation (2008), pp. 323-336.

[42] P. Mudali, T.C. Nyandeni, N. Ntlatlapay, and M.O. Adigun, "Design and Implementation of a Topology Control Scheme for Wireless Mesh Networks", IEEE AFRICON 2009.

[43] M. Gupta and S. Singh, Greening of the Internet, SIGCOMM'03, August 25-29, 2003, Karlsruhe, Germany. Copyright 2003 ACM.

[44] A. Capone and Giuliana, "Scheduling Optimization in Wireless MESH Networks with Power control and Rate Adaptation", Sensor and Ad Hoc Communications and Networks, 2006. SECON '06. (2006), 3rd Annual IEEE Communications Society. 